\title{
Induction of rapid and selective cell necrosis in Drosophila using Bacillus thuringiensis Cry toxin and its silkworm receptor
}

\author{
Fumiaki Obata $^{1 \dagger}$, Shiho Tanaka ${ }^{2 \dagger}$, Soshiro Kashio ${ }^{1}$, Hidenobu Tsujimura ${ }^{3}$, Ryoichi Sato ${ }^{2 *}$ and Masayuki Miura ${ }^{1,4^{*}}$
}

\begin{abstract}
Background: Genetic ablation of target cells is a powerful tool to study the origins and functions of cells, tissue regeneration, or pathophysiology in a human disease model in vivo. Several methods for selective cell ablation by inducing apoptosis have been established, using exogenous toxins or endogenous proapoptotic genes. However, their application is limited to cells with intact apoptotic machinery.

Results: Herein, we established a method for inducing rapid and selective cell necrosis by the pore-forming bacterial toxin Cry1Aa, which is specifically active in cells expressing the Cry1Aa receptor (CryR) derived from the silkworm Bombyx mori. We demonstrated that overexpressing CryR in Drosophila melanogaster tissues induced rapid cell death of CryR-expressing cells only, in the presence of Cry1Aa toxin. Cry/CryR system was effective against both proliferating cells in imaginal discs and polyploid postmitotic cells in the fat body. Live imaging analysis of cell ablation revealed swelling and subsequent osmotic lysis of CryR-positive cells after 30 min of incubation with Cry1Aa toxin. Osmotic cell lysis was still triggered when apoptosis, JNK activation, or autophagy was inhibited, suggesting that Cry1Aa-induced necrotic cell death occurred independently of these cellular signaling pathways. Injection of Cry1Aa into the body cavity resulted in specific ablation of CryR-expressing cells, indicating the usefulness of this method for in vivo cell ablation.
\end{abstract}

Conclusions: With Cry toxins from Bacillus thuringiensis, we developed a novel method for genetic induction of cell necrosis. Our system provides a "proteinous drill" for killing target cells through physical injury of the cell membrane, which can potentially be used to ablate any cell type in any organisms, even those that are resistant to apoptosis or JNK-dependent programmed cell death.

Keywords: Bacillus thuringiensis, Bombyx mori, Cry toxin, Drosophila melanogaster, Genetic ablation, Necrosis

\section{Background}

Cell ablation followed by phenotypic analysis is an effective method for investigating the function and origin of cells, tissue regeneration, or pathophysiology in a model of human diseases. In contrast to surgical, laser, or pharmacological ablation, genetic ablation is a reproducible, versatile, and technically accessible method for inducing death of target cells in vivo. Various genetic ablation methods have been developed. For example, direct

\footnotetext{
*Correspondence: ryoichi@cc.tuat.ac.jp; miura@mol.f.u-tokyo.ac.jp ${ }^{\dagger}$ Equal contributors

${ }^{2}$ Graduate School of Bio-Applications and Systems Engineering, Tokyo University of Agriculture and Technology, 2-24-16 Naka-cho, Koganei-shi, Tokyo 184-8588, Japan

'Department of Genetics, Graduate School of Pharmaceutical Sciences, The University of Tokyo, 7-3-1 Hongo, Bunkyo-ku, Tokyo 113-0033, Japan Full list of author information is available at the end of the article
}

induction of apoptosis by expressing modified caspase- 8 $[1,2]$, caspase-3 [3], or caspase-9 [4] under cell typespecific promoters is well established. Exogenous toxins such as the Diphtheria toxin (DT) A subunit have also been used to induce cell death by inhibiting protein synthesis $[5,6]$. Conditional expression of the DT receptor allows specific ablation of DT receptor-expressing cells by DT injection [7, 8]. Enzyme-triggered conversion of prodrugs to cytotoxic compounds is another possible strategy $[9,10]$. Although the mechanisms of cell death are different, most of these manipulations stimulate the genetically programmed process of apoptosis in the host [11].

In Drosophila melanogaster, several genetic ablation methods using DT A, Ricin A, or pro-apoptotic molecules such as Reaper, Hid, Grim (RHG) motif proteins have been established [12]. Targeted cell ablation is 
usually achieved by combining cell type-specific Gal4 drivers with cell death-inducing genes downstream of the upstream activation sequence (UAS). However, these methods are again predominantly dependent on the apoptotic machinery of target cells. Apoptosis is a timeand energy-consuming process and sometimes results in incomplete cell ablation. Unsuccessful induction of cell death can trigger diverse cellular processes due to mild activation of caspases, confusing the outcome of ablation experiments [13]. Moreover, not all types of cells are susceptible to apoptotic stimuli, as either loss or gain of function of pro- or anti-apoptotic signaling components prevents cells from undergoing apoptosis [14-16]. There is a need for a genetic technique that induces cell death in a more direct physical manner, and can therefore be applied to a wider range of cell types.

Cry toxins are proteinous insecticidal toxins produced by Bacillus thuringiensis during sporulation. Diverse families of Cry toxins exhibit toxicity in diverse but different target insects, and are thus used as environment-friendly biological pesticides that can kill the specific insect pests with few side effects for the ecosystem. Genetically modified crops producing Cry toxins have been generated, and 66 million hectares were planted worldwide in 2011 [17]. The mode of action by which Cry toxins kill specific insects is not completely understood. However, toxin-receptor interaction is regarded as an essential step for exerting insecticidal activity, as susceptible insects express Cry toxin receptors such as alkaline phosphatase, aminopeptidase $\mathrm{N}$, cadherin-like protein, or $\mathrm{ABC}$ transporter $\mathrm{C} 2$ (ABCC2) in the midgut [18]. Upon binding to receptors, Cry toxins form oligomers that generate pores in the cell membrane, leading to cell swelling and osmotic cell lysis. Previous studies have demonstrated that ectopic expression of the Bombyx mori receptors cadherin-like protein $B t R 175$ or $B m A B C C 2$ is sufficient for Cry1A toxins to induce cell death in cultured Sf9 cells, although BtR175 is less efficient [19]. Since Cry1Aa does not exhibit toxicity against non-target organisms, including Drosophila melanogaster, it can be used for conditional cell ablation if the Cry1Aa receptor is selectively expressed. In this report, we established a novel ablation method for rapid and selective induction of cell necrosis achieved by conditional expression of Cry1Aa receptors.

\section{Results}

Targeted necrosis of Cry1Aa receptor-expressing cells in cultured imaginal discs incubated with Cry1 Aa

To test whether Cry1Aa can induce cell death in cells overexpressing Cry1Aa receptors, we prepared the transgenic flies $U A S-B m A B C C 2$ and $U A S-B t R 175$. We overexpressed each receptor in the wing pouch region, which eventually develops into adult wings, using the WP-Gal4 driver [20]. These flies had no morphological defects in the wing pouch of third instar larvae or in adult wings, suggesting that the ectopic expression of exogenous Bombyx mori receptors in these cells was not toxic. We dissected and cultured wing discs from third instar larvae and treated them with Cry1Aa ex vivo. We observed propidium iodide (PI)-positive dying cells in the wing pouch region when $B m A B C C 2$, but not $B t R 175$, was overexpressed (Fig. 1a-c, Additional file 1: Figure S1). PI is a nucleic acid stain that acts as a marker for cell death, particularly for necrosis, as it enters the cells only when membrane integrity is disrupted. Since $B m A B C C 2$ is sufficient for Cry1Aa-induced cell death, we designated this receptor as CryR (Cry1Aa toxin receptor) for simplicity. We defined CrylAa-induced osmotic cell lysis as a form of necrotic cell death (or accidental cell death) that was not genetically regulated.

When CryR was driven by $d p p$-Gal4, which expresses at the midline region of wing discs, strong PI signals were observed in CryR-expressing cells when incubated with Cry1Aa (Fig. 2a, b). Simultaneous expression of both receptors further enhanced susceptibility to Cry1Aainduced cell death (Fig. 3), similar to the synergistic effect observed in Sf9 cells [19]. Serial dilutions of Cry1Aa in the culture medium (concentrations 6.25-100 nM) revealed that $12.5 \mathrm{nM}$ Cry1Aa induced weak but significant PI staining. This became stronger as the toxin concentration was increased; therefore, dose-dependent control of the strength of ablation might be possible (Additional file 1: Figure S2). These data indicate that the Cry1Aa/CryR ablation system can be used to induce conditional cell necrosis in Drosophila.

\section{Rapid and selective cell necrosis by Cry1 Aa in cultured imaginal discs and fat body}

To investigate the precise time course of Cry1Aa-induced cell necrosis, we performed live imaging analysis of cultured wing discs from WP > GFP, CryR with $200 \mathrm{nM}$ Cry1Aa (Fig. 4a, Additional files 2, 3 and 4). PI signals were first detected after around $30 \mathrm{~min}$, and increased throughout the 90-minute incubation period. Interestingly, the morphology of the wing pouch region drastically changed just before PI-positive cells were first observed. We observed swelling of GFP-positive cells, which resulted in expansion of the wing pouch region. The WPGal4 driver was also expressed in a small population of leg discs, and these cells also died by cell swelling following Cry1Aa treatment.

Imaginal discs are proliferating tissues that seem to be relatively sensitive to cell death stimuli. Therefore, we investigated whether the Cry $1 \mathrm{Aa} / \mathrm{CryR}$ ablation system was applicable to non-proliferating tissues, such as the fat body, by live imaging analysis (Fig. 4b, Additional file 5). 

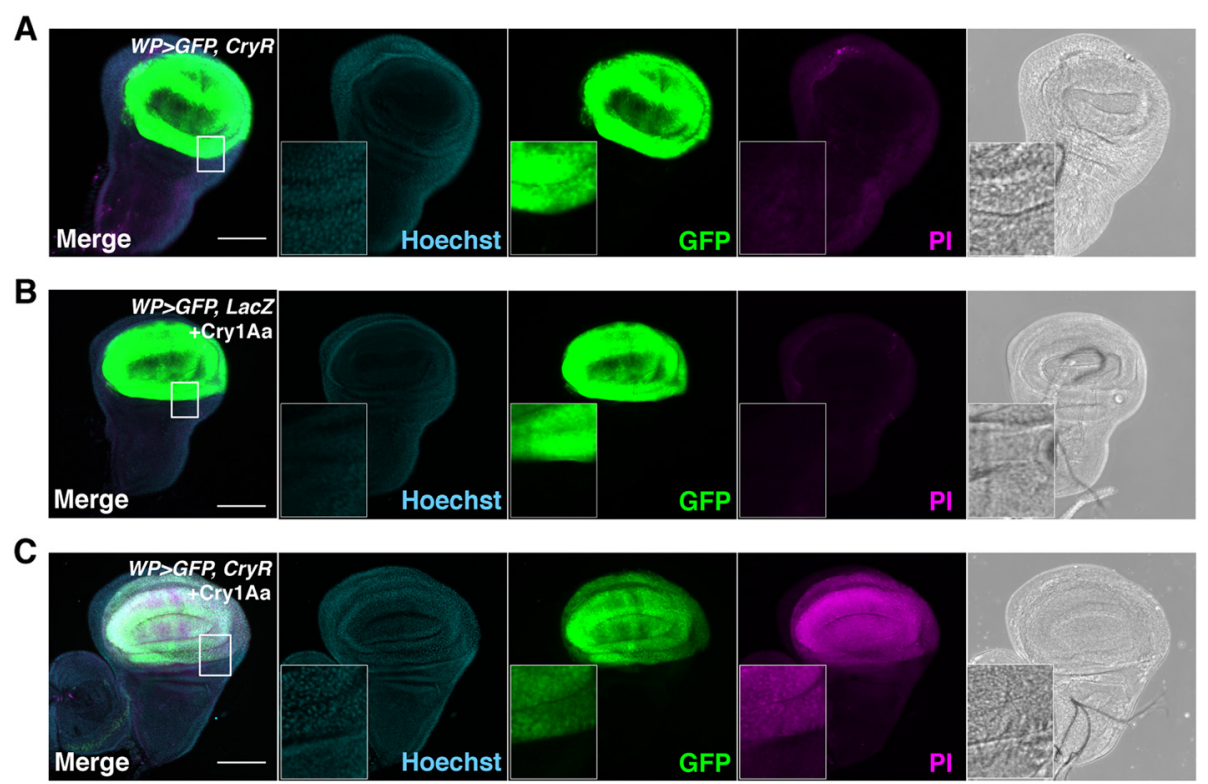

Fig. 1 Cry1Aa toxin induces cell necrosis in Cry1Aa toxin receptor (CryR)-overexpressing cells in wing pouch in wing discs. (a-c) Propidium iodide (PI) staining of cultured wing discs from third instar larvae expressing CryR by WP-Gal4. WP > GFP, CryR incubated $1 \mathrm{~h}$ with $100 \mathrm{nM}$ CrylAa showed PI signal (c), in contrast to negative controls WP > GFP, LacZ with $100 \mathrm{nM}$ Cry1Aa (b) or WP > GFP, CryR without Cry $1 \mathrm{Aa}$ (a). Scale bar, $100 \mu \mathrm{m}$

The fat body, a counterpart of mammalian liver and white adipose tissue, is composed of large, postmitotic, polyploid cells. We overexpressed CryR randomly in fat body cells using the flip-out clone technique, which simultaneously labels CryR-positive cells with GFP. When incubated with 100 nM Cry1Aa, CryR-expressing cells became PI-positive 20 min after toxin treatment, without any effect on neighboring CryR-negative cells. This suggests that the Cry1Aa/
CryR system is a highly selective method for inducing necrosis with single cell resolution.

Cry1 Aa induced necrosis independently of apoptosis, c-Jun N-terminal kinase (JNK) activation, or autophagy One of the limitations of conventional cell ablation systems is their dependency on the cell's ability to activate programmed cell death. In contrast, Cry1Aa directly
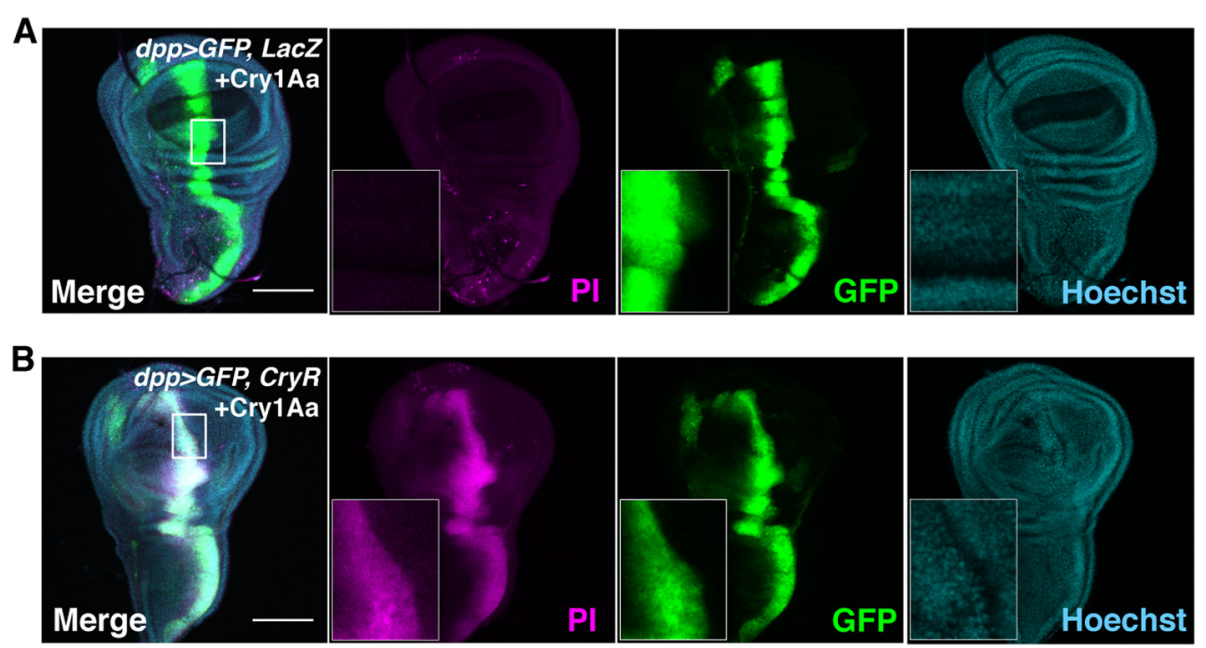

Fig. 2 Cry1Aa toxin induces cell necrosis in Cry1Aa toxin receptor (CryR)-overexpressing cells in $d p p>$ GFP, CryR wing discs. (a, b) PI staining of cultured wing discs from third instar larvae expressing CryR by dpp-Gal4.dpp > GFP, CryR incubated $1 \mathrm{~h}$ with $100 \mathrm{nM}$ CrylAa showed PI signal (b), while $d p p>$ GFP, LacZ incubated with Cry1Aa did not (a). Scale bar, $100 \mu \mathrm{m}$ 


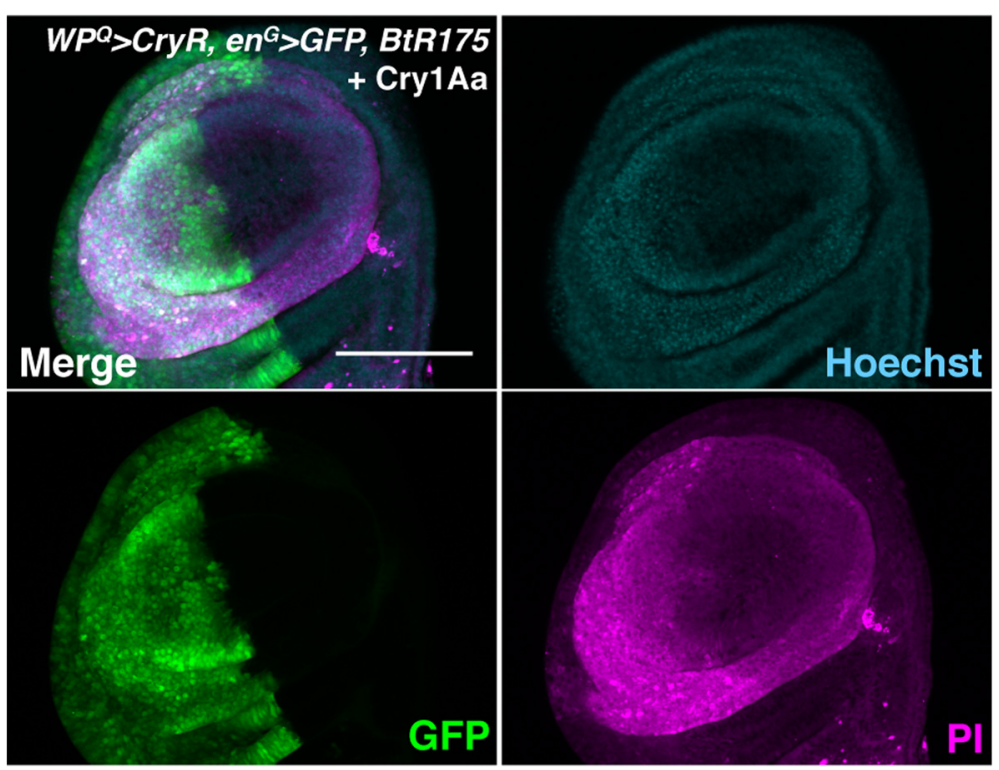

Fig. 3 Synergistic effect of simultaneous expression of CryR and BtR175 on Cry1Aa-induced toxicity. Propidium iodide (PI) staining of wing discs from third instar larvae overexpressing BtR175 by en-Gal4 and CryR by WP-QF2 incubated for $1 \mathrm{~h}$ with 50 nM Cry1Aa. Genotype is w; en-Gal4, UAS-GFP/+; WP-QF2, UAS-BtR175/QUAS-CryR. PI signals were stronger in the posterior half of the wing pouch where both BtR175 and CryR were expressed. Scale bar, $100 \mu \mathrm{m}$

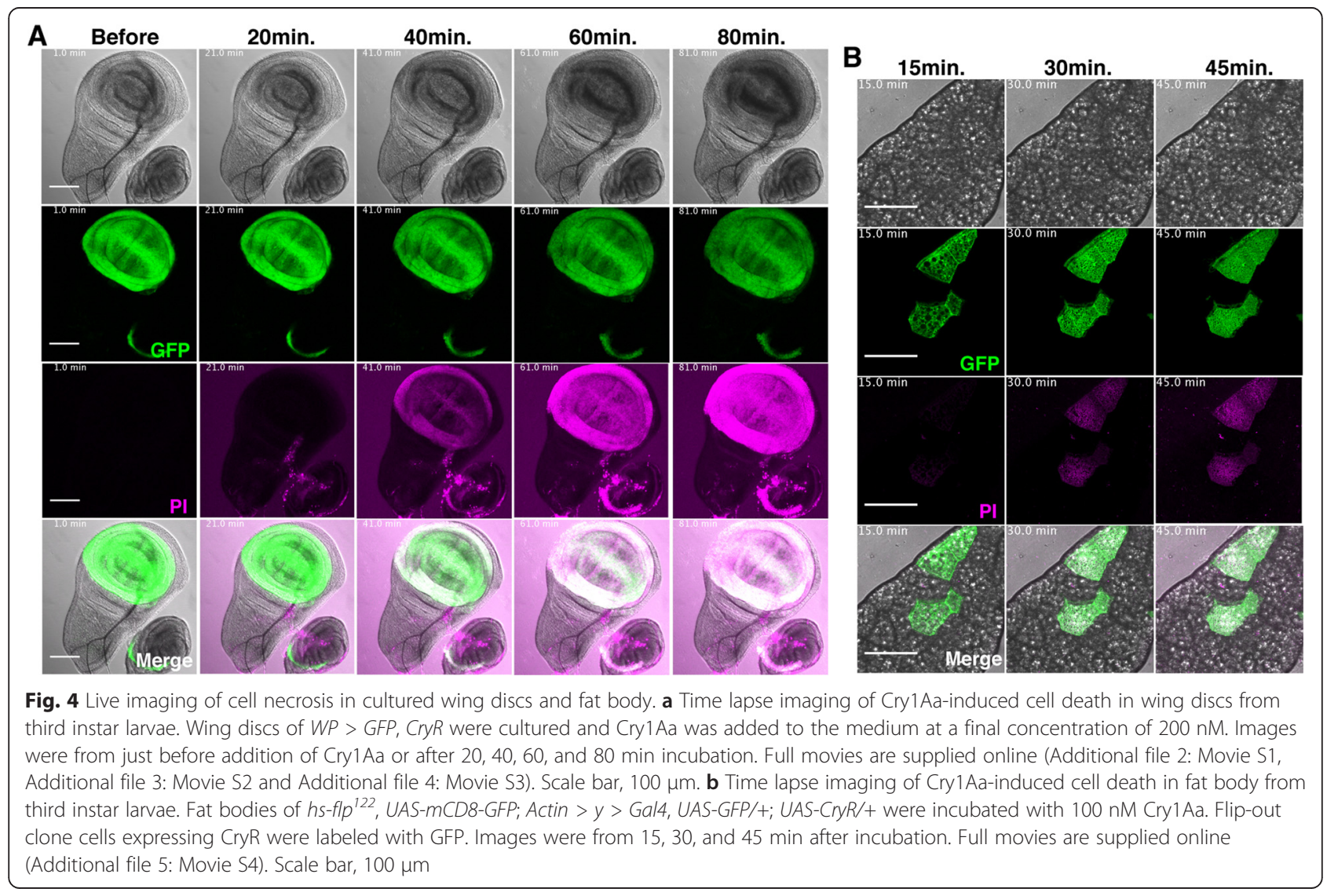


forms a pore on the plasma membrane, suggesting that cellular context does not affect toxicity. To test this, we overexpressed $p 35$, a baculoviral inhibitor of apoptosis that inactivates caspases, and tested whether Cry1Aa could still induce cell death (Fig. 5a, b). As expected, PI signals were not attenuated by p35 overexpression. In addition, Cry1Aa-induced cell death was not blocked by treatment with z-VAD-fmk, a pan-caspase inhibitor (Additional file 1: Figure S3A). We further confirmed that knock down of either dronc, an initiator caspase, or of pro-apoptotic RHG genes, did not inhibit cell death (Additional file 1: Figure S3B, C), indicating that apoptosis is not required for the Cry/CryR system.

JNK activation can also induce programmed cell death [21]. For example, Eiger, a tumor necrosis factor superfamily protein in Drosophila, kills cells in a JNKdependent manner, at least partially by a different mechanism from apoptosis. We therefore overexpressed puckered ( $p u c$ ), which is a negative regulator of JNK. Similar to $p 35$, puc expression did not affect Cry1Aa-induced cell necrosis (Fig. 5c).

Autophagic cell death is another type of programmed cell death $[22,23]$. For example, during metamorphosis in Drosophila, degeneration of larval salivary gland or midgut requires autophagic components [24, 25]. When autophagy was inhibited by knock down of atg1 alone, or together with z-VAD-fmk, we still observed PI-positive dying cells induced by Cry1Aa (Additional file 1: Figure S3D-F). These data demonstrated that Cry1Aa-induced target cell ablation does not require genetic components for cell death.

\section{Cry1 Aa injection induced selective cell necrosis in vivo}

To investigate whether the Cry1Aa/CryR system can be used for conditional cell ablation in vivo, Cry1Aa was fed to either developing larvae or adult flies through a Cry1Aa-containing diet. However, this failed to kill the animals even though $C r y R$ was overexpressed ubiquitously $(d a>C r y R)$ or in gut enterocytes $(N P 1>C r y R)$, probably due to the instability of Cry toxin in the Drosophila medium and/or in the digestive tract. Therefore, we injected concentrated Cry1Aa directly into the body cavity of wandering third instar larvae (Fig. 6a). Injection of Cry1Aa into control flies had no apparent effect on viability or development, suggesting that there is little "off-target effect" on flies without exogenous CryR. In contrast, when Cry1Aa was injected into WP >GFP, $C r y R$ larvae, we observed selective cell necrosis in the wing pouch region (Fig. 6b, c). If cells in the wing pouch undergo successful ablation, injected larvae should
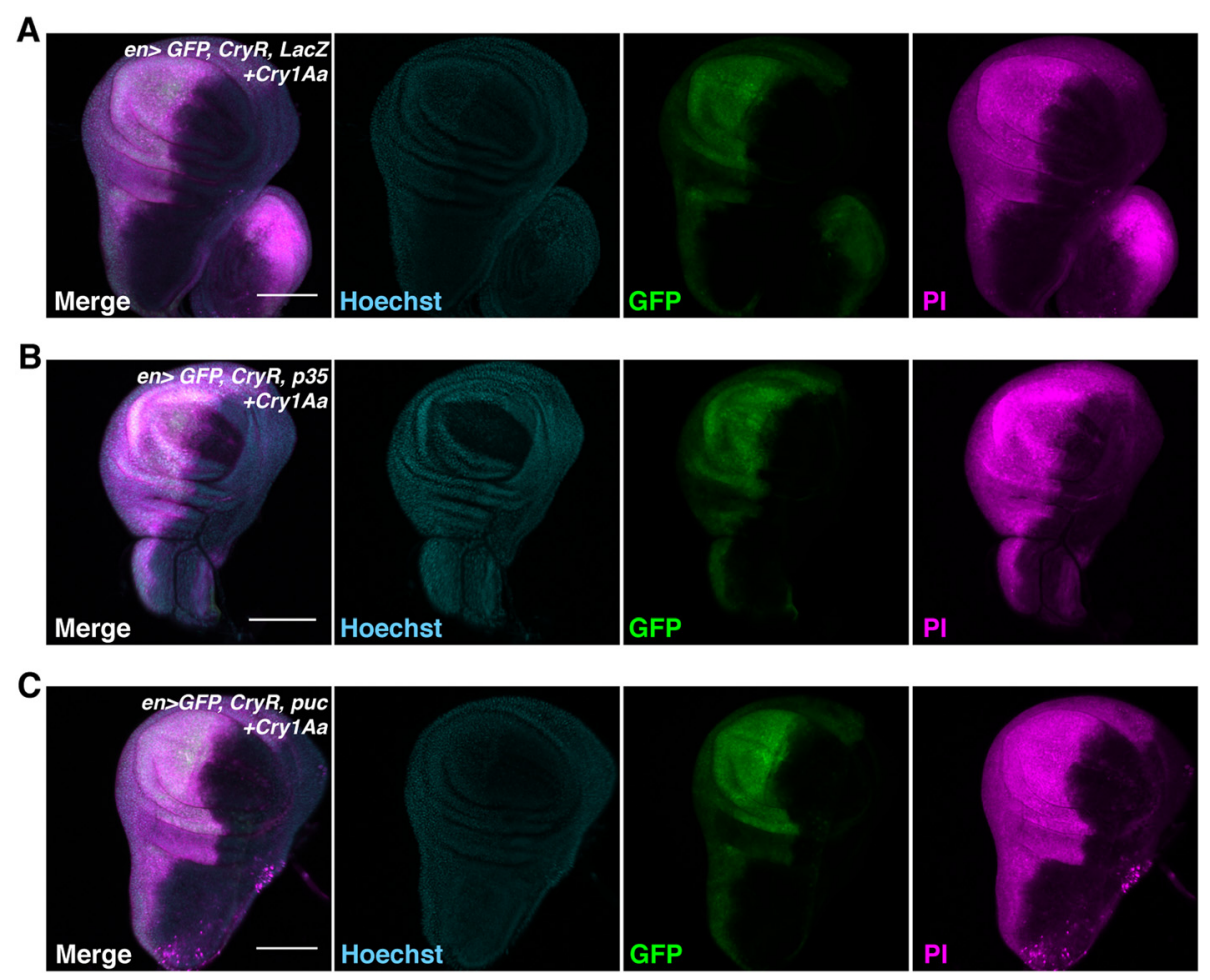

Fig. 5 Cry 1 Aa induces cell death even in cells with inhibited apoptosis or JNK-dependent cell death. (a-c) Propidium iodide (PI) staining of cultured wing discs from third instar larvae. En > GFP, CryR was crossed with LacZ (a), inhibitor of apoptosis, p35 (b), and JNK inhibitor, puC (c), and wing discs from F1 progeny were incubated for $1 \mathrm{~h}$ with $100 \mathrm{nM}$ Cry1Aa. Scale bar, $100 \mu \mathrm{m}$ 


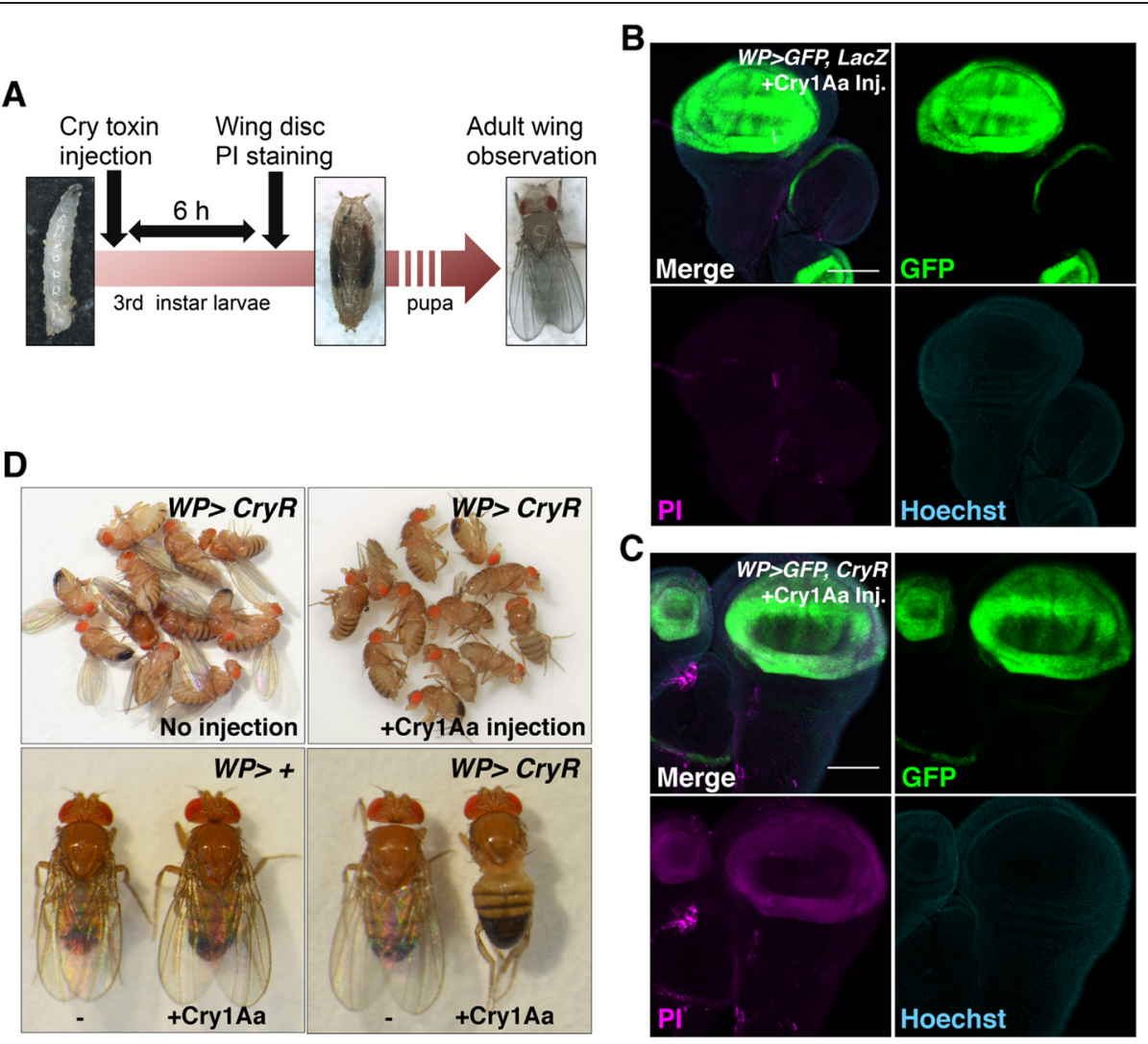

Fig. 6 Injection of Cry1Aa for in vivo cell ablation. a Schematic of an in vivo ablation experiment. (b, c) Propidium iodide (PI) staining of wing discs from Cry1Aa-injected larvae. All CryR-expressing cells, marked by green fluorescent protein (GFP), were PI-positive (c), in contrast to the controls, overexpressing LacZ (B). Scale bar, $100 \mu \mathrm{m}$. d Adult flies injected with Cry1Aa during third instar larvae lost their wings. This phenotype is not observed in the absence of either CryR or Cry1Aa injection

become adult flies without wings. Strikingly, just a single Cry1Aa injection in third instar larvae resulted in adults without wings (Fig. 6d). This phenotype was only observed in flies expressing CryR and exposed to Cry1Aa, indicating conditional cell ablation by the Cry1Aa/CryR system in vivo.

We also tested whether our system is applicable to the developmental study of sensory organs by injecting Cry1Aa toxin into Neur $>$ CryR third-instar larvae. NeurGal4 is expressed in sensory organ precursors (SOPs) in wing discs that become adult bristles. Cry1Aa injection during late-third instar larvae (6-12 h before pupal formation) resulted in a loss of bristles from epithelia (Fig. 7a). Although not all bristles are lost, the loss of bristles is probably due to differences in developmental timing as some SOPs such as the anterior scuteller bristle, which arise during later stages of development (0-6 $\mathrm{h}$ before pupal formation). Indeed, ablated macrocheates such as posterior scuteller bristle, or posterior drosocentral bristle are generated in early to middle-third larval stage (12-30 $\mathrm{h}$ before pupal formation) [26]. This suggested that Cry1Aa kills cells rapidly upon injection into larvae, and that Cry1Aa can be inactivated or removed from the hemolymph within a relatively short period after injection. Therefore, the Cry1Aa/CryR system could be useful for spatiotemporal cell ablation. Furthermore, a lack of melanization in adult epithelia at the original SOP positions implied that dying SOPs are eventually eliminated from the tissue rather than remaining in the epithelial sheet.

We observed a lethal effect of injected Cry1Aa on adult male flies with ubiquitous expression of CryR (Fig. 7b). Almost all CryR-expressing flies died within 18 $\mathrm{h}$ of the injection, while all control flies survived. In addition, Cry1Aa injection into Elav > CryR where CryR was overexpressed in neurons, resulted in an unsteady gait that eventually led to organismal death as early as 6 $\mathrm{h}$ post-injection (Additional file 6, Fig. $7 \mathrm{~b}$ ), suggesting that CryR-expressing neurons were damaged by Cry1Aa. To test whether Cry1Aa could permeate across the blood-brain barrier (BBB) and induce cell death in CNS, we performed PI staining upon Cry1Aa injection into larval hemolymphs. The injected brain from Elav $>C r y R$ larvae were positive for PI staining, but only at the surface of the brain tissue (Fig. 7c). BBB in larval brain is 
A

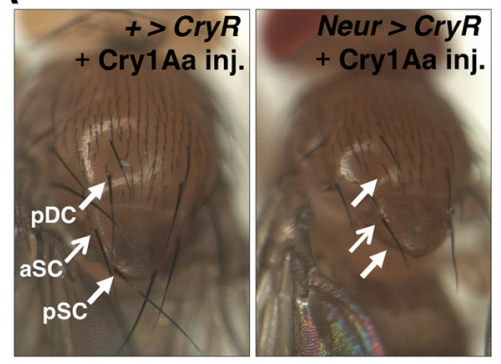

B

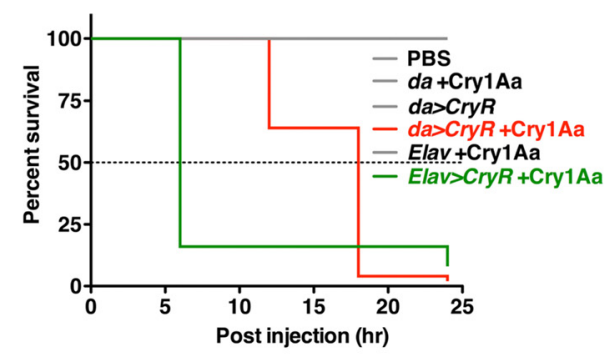

D

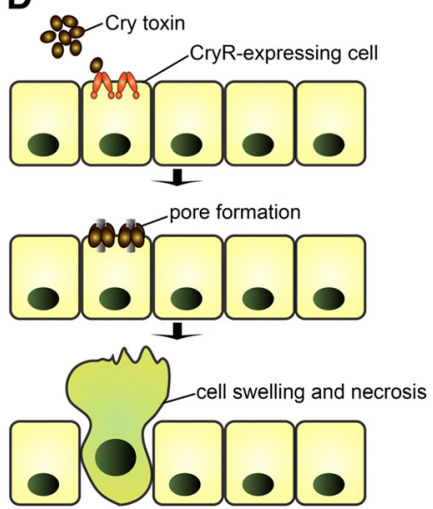

Fig. 7 In vivo cell ablation of peripheral or central nervous system by Cry1Aa injection. a Adult flies injected with Cry1Aa during third instar larval stage lost their bristles. Arrows in the left panel (negative control, without Neur-Gal4) indicate bristle positions (pDC, posterior Dorsocentral; aSC, anterior Scuteller; pSC, posteior Scuteller) and arrows in the right panel (Neur > CryR) indicate the presence (aSC) or absence (pDC, pSC) of bristles. b Survival curve of male flies with CryR expression throughout the whole body (da) or only in neurons (Elav) with and without Cry $1 \mathrm{Aa}$; $\mathrm{n}=50$ for each condition. c Confocal images of Propidium iodide (PI) staining of larval brain from Elav > CryR, with and without Cry1Aa injection. Brains were dissected $3 \mathrm{~h}$ post-injection and then stained with PI. A single focal plane from the middle and surface regions are shown. $\mathbf{d}$ Schematic view of selective cell ablation by the Cry1Aa/CryR system. Cry1Aa specifically induces cell swelling and necrosis in CryR-expressing cells by pore formation in the plasma membrane

established by surface glia, which are distinct from cortex glia $[27,28]$. To further validate that Cry1Aa cross the BBB, we overexpressed CryR by cortex glia driver, Nrv2-Gal4, and then investigated whether injected Cry1Aa into the hemolymph could induce cell death in these glia. Compared to the control (without Cry1Aa), we observed a large portion of PI-positive cortex glia, although PI-positive cells observed were not limited to GFP-positive cells (Additional file 1: Figure S4). Therefore, we believe that Cry1Aa penetrated the BBB, just as DT did in mice [8], although cells deep inside the tissue were not affected, suggesting that $\mathrm{Cry} 1 \mathrm{Aa} / \mathrm{CryR}$ system might be applicable for CNS.

\section{Discussion}

Inducing cell death of target cells is increasingly required by both basic biologists, who study the function of specific cells of interest, and clinical scientists seeking selective ablation of unwanted cells such as tumor cells. Because different types of Cry toxins and receptors have different biochemical characteristics, we can select the desired toxin-receptor combinations for each experiment or for each organism. Further, utilizing two receptors can broaden the application. For example, ablation of target cells by expressing two receptors (e.g. CryR and BtR175) simultaneously enhances cell toxicity, as observed in the present study. Moreover, highly specific ablation may be possible using relatively low concentrations of Cry1Aa and two receptors driven by different promoters, which may result in the death of only "merged" cells expressing both receptors.

Recently, Ichikawa et al. [29] developed a method for inducing reactive oxygen species production using a chemical photosensitizer, which was shown to be useful for selective cell ablation in Drosophila tissue. This probe (HMDESeR- $\beta$ Gal) is highly conditional, as it is activated by light irradiation only in cells expressing E. coli $\beta$ galactosidase. However, it is difficult to use this probe for cell ablation in internal tissues, and maintaining tissue in the dark is essential for keeping HMDESeR- $\beta$ Gal latent during the non-ablation period. Therefore, the Cry/CryR system has some advantages, especially for in vivo ablation. 
The Cry/CryR system provides spatiotemporally regulated induction of cell death. Temporal control of genetic ablation is useful for studying tissue repair or regeneration after tissue injury. Therefore, this system could be used for genetic studies of factors for tissue repair/regeneration. Furthermore, we believe that the Cry/CryR system is probably applicable to selective cell ablation in mammals, including mice, as no obvious toxicity has been observed so far. Conditional expression of CryR by the Cre/LoxP system should permit conditional ablation of mouse cells in vivo.

\section{Conclusions}

Cry toxins are widely used for pest control because of their specific insecticidal activity. Our present study demonstrates the possibility of using Cry toxins as a tool for conditional ablation in model organisms like Drosophila (Fig. 7d). Since Cry1Aa is a pore-forming toxin that physically damages the target cell membrane, it can induce necrosis in any type or condition of cells, theoretically in any species.

\section{Methods}

\section{Drosophila stocks and genetics}

Fly stocks were maintained on a standard diet containing glucose, yeast, and cornmeal at $25{ }^{\circ} \mathrm{C}$ and $60 \%$ humidity under $12 \mathrm{~h}: 12 \mathrm{~h}$ light:dark conditions. UASAtg1-RNAi (HMS02750), WP-Gal4 [20], dpp-Gal4, Nrv2Gal4, and en-Gal4 were obtained from the Bloomington Drosophila stock center. Clonal expression in the fat body was based on the flip-out technique, as described previously [20]. WP-QF2 (Kashio et al, in preparation) was constructed from a $Q F 2$ fragment gifted by CJ Potter [30]. UAS-BtR, UAS-CryR, and QUAS-CryR flies were established as described below. UAS-dronc-RNAi was established and characterized previously [20]. UAS-RHGRNA $i$ was a gift from $\mathrm{CH}$ Chen [31].

\section{Construction of CryR transgenic flies}

To generate the expression constructs $U A S-C r y R$ and $p Q U A S T-C r y R$, the full length cDNA of CryR/BmABCC2 was amplified by PCR from the pBAC4x-1-EGFPBmABCC2 vector [19] with primers (5'-CGTgcggccgc ATGAATAGTGATGGGAGAG-3' and 5'-TGAgcggccgc TTTTTCTGTATTTCTACC-3') or (5' -CGCtctagaATGA ATAGTGATGGGAGAGC-3' and 5'-CGCtctagaTCATT TTTCTGTATTTCTACCAAGATG-3'), and cloned into pUAST or pQUAST vectors using NotI or XbaI sites, respectively.

To generate the expression construct $U A S-B t R 175$, the cDNA of the toxin binding region of BtR175 [32] was obtained by PCR from the pBAC4x-1-EGFP-BtR175TBR vector [19], with primers (5'-CCGActcgagATGGG AGTTGACGTTCGAAT-3' and 5' - CCGActcgagTTA
TGCCAAATTGACAGCTA-3'), and cloned into the pUAST vector using the XhoI site.

\section{Preparation of recombinant active Cry $1 \mathrm{Aa}$}

Cry1Aa toxin was expressed as a recombinant protein in $E$. coli, and Cry1Aa protoxin was solubilized under highly alkaline conditions ( $\mathrm{pH}$ 11.0) by $\mathrm{NaOH}$. The solubilized protoxin was activated by trypsin and purified using HPLC, as described previously [33]. The concentration of the purified and activated toxin was measured by densitometry using SDS-PAGE, with bovine serum albumin as a standard.

\section{PI staining analysis of wing discs ex vivo}

For PI staining, wing discs were dissected from third instar larvae and incubated with $100 \mathrm{nM}$ (otherwise stated) nM Cry1Aa toxin, $4 \mu \mathrm{M}$ PI, and $16 \mu \mathrm{M}$ Hoechst in $\mathrm{S} 2$ medium (GIBCO) at $25{ }^{\circ} \mathrm{C}$ for $60 \mathrm{~min}$. After extensive washing in S2 medium, the wing discs were fixed with $4 \%$ PFA for $20 \mathrm{~min}$ at room temperature and then placed on glass slides. Images were obtained using a Leica SP8 confocal microscope and processed using ImageJ software.

\section{Live imaging analysis of wing discs and fat body}

Wing discs were dissected from late third instar WP > CryR larvae in S2 medium and mounted on $35 \mathrm{~mm}$ glassbottomed dishes with $50 \mu \mathrm{L}$ PBS. After removing the PBS, $500 \mu \mathrm{L} \mathrm{S} 2$ medium was added and time-lapse imaging was performed for $2 \mathrm{~h}$, at 1-min intervals, using a Leica SP5 confocal microscope. Cry1Aa was added to the culture medium at a final concentration of $200 \mathrm{nM}$ between the first and second image acquisitions. For fat body imaging, fat bodies were dissected from late third instar larvae of genotype, $h s-f l p^{122}$, UAS-mCD8-GFP; Actin > y Gal4, $U A S-G F P /+; U A S-C r y R /+$. The fat body was mounted on a glass slide with a small incubation space, with $\mathrm{S} 2$ medium containing $100 \mathrm{nM}$ Cry1Aa, and covered by cover glass. After 15-min incubation, time-lapse images were obtained using a Leica SP5 confocal microscope for $30 \mathrm{~min}$ at 1-min intervals.

\section{In vivo ablation by Cry1 Aa injection}

Cry1Aa toxin (13 ng) was directly injected into third instar larvae using the Nanoject II Auto-Nanoliter Injector (Drummond Scientific Company, Broomall, PA, USA). Wing discs were dissected $6 \mathrm{~h}$ after injection and stained with PI, or flies were maintained at $25{ }^{\circ} \mathrm{C}$ until adult hatching. Larval brains were dissected $3 \mathrm{~h}$ post-injection and stained with PI. For adult injection, male flies of da $>$ CryR or Elav $>C r y R$ were injected with $13 \mathrm{ng}$ Cry1Aa toxin using the Nanoject II Auto-Nanoliter Injector. After injection, the flies were maintained at $25{ }^{\circ} \mathrm{C}$ and dead flies were counted every $6 \mathrm{~h}$. For Elav $>$ Cry $R$ imaging, video was recorded $3 \mathrm{~h}$ after the 13-ng Cry1Aa injection. 


\section{Additional files}

Additional file 1: Figure S1. CryR, but not BtR175, is sufficient for inducing cell necrosis in Drosophila wing discs. Propidium iodide (PI) staining of wing discs from third instar larvae overexpressing BtR175, CryR, or both, with or without 100 nM Cry1Aa incubation for $1 \mathrm{~h}$. Scale bar, $100 \mu \mathrm{m}$. Figure S2. Dose-dependent response of Cry1Aa-induced cell death in wing discs. Propidium iodide (PI) staining of wing discs from third instar larvae overexpressing CryR. Wing discs were incubated for $1 \mathrm{~h}$ with 100, 50, 25, 12.5, or 6.25 nM Cry1Aa. Scale bar, $100 \mu \mathrm{m}$. Figure S3. Cry1Aa induces cell death in Drosophila wing discs in the absence of components for autophagy and apoptosis. Propidium iodide (PI) staining of wing discs from third instar larvae overexpressing CryR in their wing pouch. Discs were incubated with $100 \mathrm{nM}$ Cry1 Aa for 1 h. (A) Wing discs were cultured with $100 \mathrm{nM}$ Cry1 Aa in the presence of $200 \mu \mathrm{M}$ pan-caspase inhibitor, z-VAD-fmk. (B, C, D) Knock down apoptotic (B, C) or autophagic (D) components within the wing pouch did not suppress Cry1Aa-induced cell death. Dronc is an initiator caspase. RHG stands for pro-apoptotic genes, Reaper, Hid, and Grim (triple RNAi). (E, F) Simultaneous inhibition of apoptosis by $200 \mu \mathrm{M}$ z-VAD-fmk and autophagy by Atg1-RNAi in the presence (E) or absence (F) of 100 nM Cry1Aa. Scale bar, $100 \mu \mathrm{m}$. Figure S4. Cry $1 \mathrm{Aa}$ induces cell death of cortex glia in larval brain in vivo. Propidium iodide (PI) staining of brains from third instar larvae that overexpressed CryR by Nrv2-Gal4, a cortex glia driver. (A-C) Cry1Aa was injected during the third larval stage and brains were dissected $3 \mathrm{~h}$ after injection. (A) No Cry1Aa injection as a negative control. Genotype; Nrv2>mGFP, CryR Scale bar, $100 \mu \mathrm{m}$. (C) High magnification image of Cry1Aa injected brain. Scale bar, $20 \mu \mathrm{m}$.

Additional file 2: Live imaging of Cry $1 \mathrm{Aa}$ induced cell death in wing disc from WP>GFP, CryR. GFP (green) and PI (magenda) images.

Additional file 3: Live imaging of Cry1Aa induced cell death in wing disc from WP>GFP, CryR. Merged (GFP and PI) images.

Additional file 4: Live imaging of Cry1Aa induced cell death in wing disc from WP>GFP, CryR. Bright field images.

Additional file 5: Live imaging of Cry $1 \mathrm{Aa}$ induced cell death in fat body with CryR- and GFP-overexpressing flip-out clones. PI (magenda) and merged (PI, GFP, and bright field) images.

Additional file 6: Movies of Elav>CryR adult flies after Cry1Aa injection.

\section{Abbreviations}

ABCC2: ABC transporter C2; BBB: Blood-brain barrier; CryR: Cry1Aa toxin receptor; DT: Diphtheria toxin; JNK: c-Jun N-terminal kinase; Pl: Propidium iodide; RHG: Reaper, Hid, Grim; SOP: Sensory organ precursor; UAS: Upstream activation sequence.

\section{Competing interests}

The authors declare that they have no competing interests.

\section{Authors' contributions}

FO, ST, HT, RS, and MM designed the study. FO, ST, and SK performed experiments and data analysis. FO, ST, and MM wrote the manuscript. All authors read and approved the final manuscript.

\section{Acknowledgments}

We thank T Nishimura, N Okamoto, CH Chen, T Uemura, CJ Potter, YP Wairkar, and Bloomington Drosophila stock center for providing fly stocks and reagents. This work was supported by grants from Core Research for Evolutional Science and Technology, Japan Science and Technology Agency (to MM), Grant-in-Aid for Scientific Research (S) (23229002) (to MM), Grant-in-Aid for Scientific Research (B) (24310054) (to RS) and Grant-in-Aid for JSPS Fellows (258207) (to ST).

\section{Author details}

'Department of Genetics, Graduate School of Pharmaceutical Sciences, The University of Tokyo, 7-3-1 Hongo, Bunkyo-ku, Tokyo 113-0033, Japan. ${ }^{2}$ Graduate School of Bio-Applications and Systems Engineering, Tokyo University of Agriculture and Technology, 2-24-16 Naka-cho, Koganei-shi, Tokyo 184-8588, Japan. ${ }^{3}$ Developmental Biology, Tokyo University of Agriculture and Technology, 3-5-8 Saiwai-cho, Fuchu-shi, Tokyo 183-8509,
Japan. ${ }^{4}$ CREST, Japan Agency for Medical Research and Development, 20F Yomiuri Shimbun Bldg. 1-7-1 Otemachi, Chiyoda-ku, Tokyo 100-0004, Japan.

Received: 5 June 2015 Accepted: 19 June 2015

Published online: 08 July 2015

\section{References}

1. Carlotti F, Zaldumbide A, Martin P, Boulukos KE, Hoeben RC, Pognonec P. Development of an inducible suicide gene system based on human caspase 8. Cancer Gene Ther. 2005;12:627-39.

2. Chu Y, Senghaas N, Köster RW, Wurst W, Kühn R. Novel caspase-suicide proteins for tamoxifen-inducible apoptosis. Genesis. 2008;46:530-6.

3. Mallet VO, Mitchell C, Guidotti J-E, Jaffray P, Fabre M, Spencer D, et al. Conditional cell ablation by tight control of caspase-3 dimerization in transgenic mice. Nat Biotechnol. 2002;20:1234-9.

4. Nör JE, Hu Y, Song W, Spencer DM, Núñez G. Ablation of microvessels in vivo upon dimerization of iCaspase-9. Gene Ther. 2002;9:444-51.

5. Breitman ML, Clapoff S, Rossant J, Tsui LC, Glode LM, Maxwell IH, et al. Genetic ablation: targeted expression of a toxin gene causes microphthalmia in transgenic mice. Science. 1987;238:1563-5.

6. Palmiter RD, Behringer RR, Quaife CJ, Maxwell F, Maxwell $H$, Brinster RL. Cell lineage ablation in transgenic mice by cell-specific expression of a toxin gene. Cell. 1987;50:435-43.

7. Saito M, Iwawaki T, Taya C, Yonekawa H, Noda M, Inui Y, et al. Diphtheria toxin receptor-mediated conditional and targeted cell ablation in transgenic mice. Nat Biotechnol. 2001;19:746-50.

8. Buch T, Heppner FL, Tertilt C, Heinen TJAJ, Kremer M, Wunderlich FT, et al. A Cre-inducible diphtheria toxin receptor mediates cell lineage ablation after toxin administration. Nat Methods. 2005;2:419-26.

9. Clark AJ, Iwobi M, Cui W, Crompton M, Harold G, Hobbs S, et al. Selective cell ablation in transgenic mice expression E. coli nitroreductase. Gene Ther. 1997:4:101-10.

10. Borrelli E, Heyman RA, Arias C, Sawchenko PE, Evans RM. Transgenic mice with inducible dwarfism. Nature. 1989;339:538-41.

11. Fuchs $Y$, Steller H. Programmed cell death in animal development and disease. Cell. 2011;147:742-58.

12. Sweeney ST, Hidalgo A, de Belle JS, Keshishian H. Genetic systems for functional cell ablation in Drosophila. Cold Spring Harb Protoc. 2012;2012:950-6.

13. Miura M. Apoptotic and nonapoptotic caspase functions in animal development. Cold Spring Harb Perspect Biol 2012;4(10). doi: 10.1101/ cshperspect.a008664.

14. Yin VP, Thummel CS, Bashirullah A. Down-regulation of inhibitor of apoptosis levels provides competence for steroid-triggered cell death. J Cell Biol. 2007:178:85-92.

15. Zhang Y, Lin N, Carroll PM, Chan G, Guan B, Xiao H, et al. Epigenetic blocking of an enhancer region controls irradiation-induced proapoptotic gene expression in Drosophila embryos. Dev Cell. 2008;14:481-93.

16. Hassel C, Zhang B, Dixon M, Calvi BR. Induction of endocycles represses apoptosis independently of differentiation and predisposes cells to genome instability. Development. 2014;141:112-23.

17. Tabashnik BE, Brévault T, Carrière $Y$. Insect resistance to Bt crops: lessons from the first billion acres. Nat Biotechnol. 2013;31:510-21.

18. Pardo-López L, Soberón M, Bravo A. Bacillus thuringiensis insecticida three-domain Cry toxins: mode of action, insect resistance and consequences for crop protection. FEMS Microbiol Rev. 2013;37:3-22.

19. Tanaka S, Miyamoto K, Noda H, Jurat-Fuentes JL, Yoshizawa Y, Endo H, et al. The ATP-binding cassette transporter subfamily C member 2 in Bombyx mori larvae is a functional receptor for Cry toxins from Bacillus thuringiensis. FEBS J. 2013;280:1782-94

20. Obata F, Kuranaga E, Tomioka K, Ming M, Takeishi A, Chen C-H, et al. Necrosis-driven systemic immune response alters SAM metabolism through the FOXO-GNMT axis. Cell Rep. 2014;7:821-33.

21. Igaki T, Kanda H, Yamamoto-Goto Y, Kanuka H, Kuranaga E, Aigaki T, et al. Eiger, a TNF superfamily ligand that triggers the Drosophila JNK pathway. EMBO J. 2002;21:3009-18.

22. Tsujimoto Y, Shimizu S. Another way to die: autophagic programmed cell death. Cell Death Differ. 2005;12:1528-34.

23. Yuan J, Kroemer G. Alternative cell death mechanisms in development and beyond. Genes Dev. 2010;24:2592-602. 
24. Berry DL, Baehrecke EH. Growth arrest and autophagy are required for salivary gland cell degradation in Drosophila. Cell. 2007;131:1137-48.

25. Denton D, Shravage B, Simin R, Mills K, Berry DL, Baehrecke EH, et al. Autophagy, not apoptosis, is essential for midgut cell death in Drosophila. Curr Biol. 2009;19:1741-6.

26. Huang F, Dambly-Chaudière $C$, Ghysen $A$. The emergence of sense organs in the wing disc of Drosophila. Development. 1991;111:1087-95.

27. Pereanu W, Shy D, Hartenstein V. Morphogenesis and proliferation of the larval brain glia in Drosophila. Dev Biol. 2005;283:191-203.

28. Mayer F, Mayer N, Chinn L, Pinsonneault RL, Kroetz D, Bainton RJ. Evolutionary conservation of vertebrate blood-brain barrier chemoprotective mechanisms in Drosophila. J Neurosci. 2009;29:3538-50.

29. Ichikawa Y, Kamiya M, Obata F, Miura M, Terai T, Komatsu T, et al. Selective ablation of $\beta$-galactosidase-expressing cells with a rationally designed activatable photosensitizer. Angew Chemie - Int Ed. 2014;53:6772-5.

30. Riabinina O, Luginbuhl D, Marr E, Liu S, Wu MN, Luo L, et al. Improved and expanded Q-system reagents for genetic manipulations. Nat Methods. 2015;12:219-22.

31. Siegrist $\mathrm{SE}$, Haque NS, Chen C-H, Hay BA, Hariharan IK. Inactivation of both Foxo and reaper promotes long-term adult neurogenesis in Drosophila. Curr Biol. 2010;20:643-8.

32. Nagamatsu Y, Koike T, Sasaki K, Yoshimoto A, Furukawa Y. The cadherin-like protein is essential to specificity determination and cytotoxic action of the Bacillus thuringiensis insecticidal CrylAa toxin. FEBS Lett. 1999:460:385-90.

33. Obata F, Kitami M, Inoue Y, Atsumi S, Yoshizawa Y, Sato R. Analysis of the region for receptor binding and triggering of oligomerization on Bacillus thuringiensis Cry1Aa toxin. FEBS J. 2009;276:5949-59.

\section{Submit your next manuscript to BioMed Central and take full advantage of:}

- Convenient online submission

- Thorough peer review

- No space constraints or color figure charges

- Immediate publication on acceptance

- Inclusion in PubMed, CAS, Scopus and Google Scholar

- Research which is freely available for redistribution 